\title{
A clinical profile of inpatient admissions to the psychogeriatric unit at Stikland Hospital
}

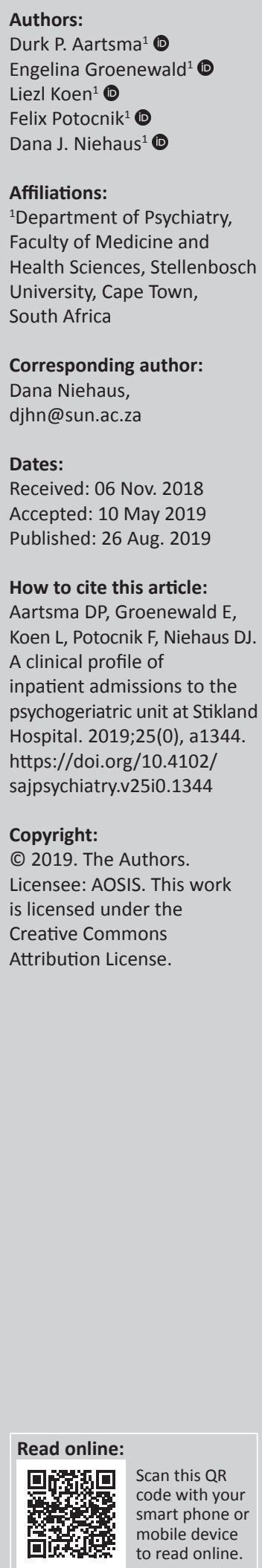

Background: Globally, the number of older people is rising. As a consequence of greater longevity, an increased burden on both medical and mental health care is expected. As a first step towards developing strategies to provide quality mental health care for this growing population, practitioners need to have a thorough understanding of the composition and needs of these patients.

Aim: To profile the inpatient population of a psychogeriatric unit in terms of demographics, diagnostic makeup, average length of stay and selected outcomes.

Setting: This study was conducted at the psychogeriatric unit of Stikland Hospital, Western Cape, South Africa.

Methods: Demographic and clinical data were retrospectively collected from patient files, discharge summaries and an admission database over a 3-year period.

Results: A total of 903 patients were referred to Stikland Hospital during a 3-year period. Of the 498 patients who were admitted, 56 were readmissions. The mean age of patients was 67 years, and more than $57 \%$ of patients were female. The majority of patients $(97.1 \%)$ were admitted as involuntary mental health users. The diagnosis of a cognitive disorder was made in $49.5 \%$ of admissions followed by psychotic disorders in $36.9 \%$ and mood disorders in $23.2 \%$. The median length of stay was 53 days.

Conclusion: The findings of this study illustrate that mental health services for the elderly in the Western Cape are insufficient, as only patients with severe illness and comorbidity could be admitted. The study emphasises the need for the restructuring of resources and the implementation of strategies, which may decrease the frequency of admissions to inpatient geriatric units.

Keywords: Elderly; Old Age Psychiatry; Geriatric; Psychogeriatric; Dementia; Neurocognitive Disorders; Inpatient.

\section{Introduction}

In $2015,12 \%$ of the world's population was more than 60 years old. It is expected that this number will rise to $22 \%$ by $2050 .{ }^{1}$ The ageing of the global population represents a major mental health concern because mental disorders such as neurocognitive disorders, depression, anxiety disorders and substance disorders become more prevalent with increasing age. ${ }^{2}$ Presently, almost $20 \%$ of people over the age of 60 suffer from a mental disorder, ${ }^{2}$ and by 2030, this number will double. ${ }^{3}$ Major neurocognitive disorder (dementia) is one of the most prevalent mental disorders in the elderly, and in 2017, 50 million people were living with this disorder globally. ${ }^{2}$ This number is expected to increase to 82 million by 2030 and 152 million by $2050 .{ }^{2}$ Depression is also common in the elderly with prevalence estimates of $7 \%$ in the general elderly population, ${ }^{2} 6 \%-10 \%$ in primary care settings and $30 \%$ in medical inpatients and long-term care settings. ${ }^{3}$. Mental disorders cause significant impairment in the quality of life of elderly patients, their caregivers and their family members. ${ }^{2}$ These disorders also exacerbate the disability associated with other medical conditions and result in increased utilisation of health services. ${ }^{2,3}$

This demographic transition is not unique to high-income countries. ${ }^{3,4}$ It is expected that $80 \%$ of the elderly will live in low- and middle-income countries by $2050,{ }^{1}$ and the greatest burden of dementia is concentrated in these countries. ${ }^{2}$ Similarly, in South Africa, the number of the population older than the age of 60 increased from 2.8 million ( $7.1 \%$ of the population) in 1996 to 4.1 million ( $8 \%$ of the population) in 2011. It is projected that this number will continue to rise and that approximately 7 million people will be older than 60 in $2030 .^{5}$ 
The prevalence of psychiatric disorders in South African elderly is nevertheless unknown. The South African Stress and Health (SASH) study estimated the lifetime prevalence of any psychiatric disorder in adults older than 65 years to be $27.9 \% .^{6}$ The lifetime prevalence of anxiety disorders was $17 \%$, mood disorders was $6.5 \%$, and substance disorders was $11 \%$. However, the 12-month prevalence of these disorders was not estimated in adults older than 65 , and the prevalence of other psychiatric disorders was not investigated in this study. Research on the prevalence of dementia in South Africa is also limited. ${ }^{7}$ The only large community prevalence study to date estimated an $8 \%$ prevalence rate for dementia in elderly people living in rural Eastern Cape. ${ }^{8}$ Other studies which quantified the prevalence of dementia in South Africa were either very small ${ }^{9,10}$ or based only on clinical samples. ${ }^{11,12}$

South Africa does not have adequate resources to treat the elderly with mental disorders. South Africa has $<5$ old-age psychiatrists and only 1 psychogeriatric training unit to cater for a current population of 57.7 million people. ${ }^{13,14}$

The growing elderly population places an increasingly greater demand on over-burdened and under-resourced inpatient psychogeriatric services. A thorough understanding of the patient population using these services is necessary to inform clinical decision-making, focus service development and to make timely provisions for the predicted needs of the elderly. ${ }^{15}$ The aim of this study was to profile geriatric inpatients at Stikland Hospital. It is expected that an insight into the clinical, socio-demographic and readmission characteristics of this age group will reflect on the service requirements and serve as a first step to improving care and accessibility of treatment for the elderly in South Africa.

\section{Methods}

\section{Study design}

We conducted a retrospective record review of patients admitted to the psychogeriatric inpatient unit at Stikland Hospital between 01 January 2013 and 31 December 2015.

\section{Study setting}

Stikland Hospital is a public, tertiary psychiatric hospital situated in the Western Cape Province, which has a total population of more than 6 million people, including 594266 persons older than $60 .{ }^{14}$ The province is divided into two catchment areas which are serviced by two tertiary academic hospitals, Groote Schuur and Tygerberg Hospital. Stikland Hospital is the only geriatric psychiatry public sector-funded inpatient unit, and it takes referrals from across the entire province.

The psychogeriatric unit at Stikland Hospital consists of three wards with a total bed capacity of 77 (33 male beds and 44 female beds). Once a referral has been reviewed and the documentation is found to be in order, the patient is added to the waiting list. The required documentation is a referral letter and correctly completed Mental Health Care Act (MHCA) forms. As soon as a patient is discharged and a bed becomes available, the first patient on the waiting list is accepted for admission.

\section{Study population}

The records of all patients admitted between 01 January 2013 and 31 December 2015 were included in the study. Patients younger than 60 years of age were excluded from the study.

\section{Data abstraction}

The following information were extracted by the principle investigator and subsequently collated on an anonymised Microsoft Excel spreadsheet:

\section{Admission data}

Admission status according to the Mental Health Care Act 17 of 2002, the name of the referring hospital, the date of referral, the date that all administrative documents were in order, the date that a patient was put on the waiting list, the date of admission and discharge, the number of admissions during the 3-year review period and the age of the patient at the first admission during this study period.

\section{Demographic data: Age and gender}

Diagnoses: Primary psychiatric diagnoses, as well as medical and/or psychiatric comorbidities, were recorded. The first psychiatric diagnosis listed on the discharge summary was considered the primary diagnosis and subsequent diagnoses were recorded as comorbid conditions. Psychiatric disorders were diagnosed according to the Diagnostic and Statistical Manual of Mental Disorders 4th edition, Text Revision (DSMIV, TR). ${ }^{16}$ The primary psychiatric diagnosis was grouped into one of four diagnostic categories, as summarised in Table 1.

\section{Analysis of data}

Continuous variables were summarised as mean \pm standard deviation (s.d.), or median and standard deviation. Nominal variables were summarised as counts and percentages. Group differences were tested using Student's $t$-test (or the Mann-Whitney U-tests for non-parametric data) and Chi-square tests. All analyses were performed using SPSS, version 25, and statistically significant differences were established at $p<0.05$.

TABLE 1: Major categories used for diagnosis of psychiatric disorders according to the Diagnostic and Statistical Manual of Mental Disorders 4th edition, text revision.

\begin{tabular}{ll}
\hline Diagnostic category & Disorder type \\
\hline Mood disorders & $\begin{array}{l}\text { Major depressive disorder, bipolar disorder, dysthymic } \\
\text { disorder, cyclothymic disorder, mood disorder due to a } \\
\text { general medical condition, substance-induced mood disorder }\end{array}$ \\
Psychotic disorders & $\begin{array}{l}\text { Schizophrenia, Schizoaffective disorder, Psychotic disorder } \\
\text { due to a general medical condition, substance-induced } \\
\text { psychotic disorder }\end{array}$ \\
$\begin{array}{l}\text { Dementia of the Alzheimer's type, vascular dementia, } \\
\text { dementia due to a general medical condition, substance- } \\
\text { induced persisting dementia, dementia due to multiple } \\
\text { aetiologies, dementia not otherwise specified }\end{array}$ \\
conditions & $\begin{array}{l}\text { Anxiety disorders, substance-related disorders, adjustment } \\
\text { disorders }\end{array}$ \\
\hline
\end{tabular}




\section{Ethical consideration}

The study was approved by the Health Research Ethics Committee of Stellenbosch University (study number: S14/08/163), as well as the Head of Establishment (Stikland Hospital). A waiver of informed consent was granted for this retrospective study. All identifiable patient information was anonymised.

\section{Results}

During the 3-year study period, 903 referrals were placed on the waiting list of which one was discovered later to be 54 years of age. We, therefore, report only on 902 referrals. $491(54.4 \%)$ of the referred patients were admitted between the ages of 60 and 89 (mean age 66.92 years, s.d. 5.93). More females than males were admitted over the period. Eightyeight $(17.9 \%)$ of the admitted patients had one to four readmissions during this period, and $54(6 \%)$ patients were still in hospital $(n=46)$ or on the waiting list $(n=8)$ by the end of the study period. The mean waiting time from being added to the waiting list until admission to the unit was 18 days, and females waited longer (22 days, s.d. 17.74) than males (14 days, s.d. 11.61; $p<0.0001$ ). The mean time spent waiting for admission increased significantly $(p<0.0001)$ from 12 days in 2013 to 22 days in 2014 and 22 days in 2015.

The number of referrals from the two catchment areas was similar. The Groote Schuur area referred 443 patients (49.1\%), of which $238(52.6 \%)$ were admitted, whereas the Tygerberg area referred 459 patients (50.9\%) of which 258 (56.2\%) were admitted. There were no significant differences in terms of percentage admitted from the waiting list ( $p=0.285$ Chi-sq). Groote Schuur Hospital itself sent the most (23.3\%) referral letters $(n=210)$ and also had the highest number of admissions $(11.8 \%, n=106)$, followed by Karl Bremer Hospital (11.1\%), Victoria Hospital (8.9\%) and New Somerset Hospital (8.0\%) during the study period.

The median length of stay was 53 days (mean 70.71, s.d. 63.1, range 1-552 days) and was not significantly influenced by age ( $p=0.707)$ or gender $(p=0.946)$. The primary diagnosis and the presence of comorbid medical and psychiatric disorders were not significantly associated with length of stay.

The number of readmissions over the 3 years varied from one to four admissions, but most patients $(88.8 \%)$ were admitted only once. Female patients had a slightly higher readmission frequency compared to males.

Almost all patients $(97.1 \%)$ were admitted as involuntary patients under the Mental Health Care Act 17 of 2002.

The most common single major psychiatric disorder diagnoses in both males and females were cognitive disorders (24.8\%), followed by psychotic disorders (23.4\%) and mood disorders $(10.6 \%)$ (Table 2). As part of a single or comorbid diagnosis, cognitive disorders remained the most common with $49.5 \%$ of cases $(n=243)$, while psychotic disorders were part of the diagnosis in $36.9 \%(n=181)$, mood disorders in $23.2 \%(n=114)$
TABLE 2: Diagnostic categories and comorbid psychiatric conditions of patients $(n=491) \dagger$

\begin{tabular}{lcc}
\hline Characteristic & Overall $(\boldsymbol{n})$ & $\mathbf{\%}$ \\
\hline Diagnostic categories & & \\
Cognitive disorder only & 122 & 24.8 \\
Cognitive disorder and anxiety disorder & 2 & 0.4 \\
Cognitive disorder and substance-related disorder & 18 & 3.7 \\
Cognitive disorder and mood disorder & 46 & 9.4 \\
Cognitive disorder and mood disorder and & 4 & 0.8 \\
substance-related disorder & & \\
Cognitive disorder and psychotic disorder & 46 & 9.4 \\
Cognitive disorder and psychotic disorder and & 5 & 1.0 \\
substance-related disorder & & \\
Psychotic disorder & 115 & 23.4 \\
Psychotic disorders and substance-related disorder & 15 & 3.1 \\
Mood disorder & 52 & 10.6 \\
Mood disorder and substance-related disorder & 9 & 1.8 \\
Mood disorder and anxiety disorder & 3 & 0.6 \\
Substance-related disorder & 2 & 0.4 \\
Eating disorders & 1 & 0.2 \\
Other & 51 & 10.4 \\
\hline
\end{tabular}

$\dagger$, Patients that were readmitted were counted as separate cases.

TABLE 3: Frequency of the most prevalent medical comorbidities.

\begin{tabular}{lcc}
\hline Variable & Frequency $(\boldsymbol{n})$ & $\mathbf{\%}$ \\
\hline Medical comorbidities & & \\
Hypertension & 208 & 47.4 \\
Hypercholesterolemia & 91 & 20.7 \\
Diabetes mellitus & 64 & 14.6 \\
Vitamin B12 deficiency & 43 & 9.8 \\
Chronic obstructive pulmonary disease & 31 & 7.1 \\
Hypothyroidism & 26 & 5.9 \\
\hline
\end{tabular}

and substance-related disorders in $10.8 \%(n=53)$. The most frequent psychiatric comorbid diagnoses were substancerelated disorders and Vascular Dementia $(n=79)$ and substance-related disorders $(n=51)$. Of the 243 patients with cognitive disorders, the most common types of cognitive disorders were Vascular Dementia $(63.4 \%, n=154)$, Dementia Not Otherwise Specified $(19.8 \%, n=48)$ and Dementia Due to Multiple Aetiologies $(8.6 \%, n=21) .5 .8 \%$ of patients $(n=14)$ had Dementia of the Alzheimer's Type and 2.5\% $(n=6)$ had Dementia Due to a General Medical Condition.

There was a high prevalence $(74.5 \%)$ of medical comorbidities within this population (Table 3). The number of medical comorbidities ranged from 1 to $4(1=27.5 \%, 2=25.3 \%$, $3=13.6 \%, 4=7.9 \%)$ and did not impact the length of stay $(p=\mathrm{NS})$. Hypertension and hypercholesterolemia were the most prevalent medical comorbidities in this sample (Table 3).

\section{Discussion}

This 3-year retrospective review of admissions to a psychogeriatric unit in the Western Cape provides a broad overview of patient characteristics and emphasises the array of psychiatric disorders and comorbidities affecting this population.

\section{Admissions}

The Stikland Hospital psychogeriatric unit is the only specialised inpatient unit for elderly patients with psychiatric 
disorders in the Western Cape. Yet, only approximately half of the patients who required further inpatient care at the unit were admitted during this time period. Of the 411 patients on the waiting list who were not admitted, only 8 remained on the waiting list at the end of the study. The remaining 403 patients were probably discharged from the referring hospitals due to bed pressure, and improvement in their symptoms while they were waiting for admission to Stikland Hospital.

The time that patients had to wait for admission to the unit increased markedly from 2013 to 2015. The actual waiting time is probably much longer than the period demonstrated in this study (22 days in 2015) as patients were only added to the list once the referring agent provided the correct referral documents. Females waited significantly longer than males, which could be explained by the fact that the unit does not have an adequate number of female beds. It is apparent from these findings that the Stikland psychogeriatric unit is unable to meet the increasing demand for psychogeriatric services in the Western Cape Province.

The median length of stay of 53 days is much longer than similar international studies (median 28 and 29 days) ${ }^{15,17}$ Due to the complexity of psychiatric conditions in the elderly, these patients often require a longer hospital admission. Similar international studies demonstrated a strong association between the number of admissions, the length of stay and the psychiatric diagnosis: psychotic and mood disorders required longer and more frequent admissions than other psychiatric conditions. ${ }^{15,17}$ These associations were not demonstrated in our study.

Involuntary care was required in the majority of patients (97.1\%). This number is much higher than in psychogeriatric units in developed countries ${ }^{15}$ and the general adult psychiatric population in South Africa $(75.6 \%) .{ }^{18}$ The high number of involuntary admissions to our unit indicates that the unit mostly admitted patients with a severe mental illness, who posed a high risk to themselves and others. Patients who could consent to admission (assisted and voluntary patients) did not get the opportunity to be admitted to the psychogeriatric unit due to the shortage of beds.

\section{Demographic information}

The mean age of patients in this study was 66.92 years. This is much lower than a similar study conducted in Australia ${ }^{15}$ where the mean age of 516 patients admitted to an aged psychiatry facility was 74.7 years. The lower mean age in our study could be explained by the fact that the majority of South African elderly is between 60 and 64 years old, and that South Africa has a much lower median population age than many high-income countries. ${ }^{5}$ Similar to our study, a high proportion of patients in the Australian study were female. ${ }^{15}$ The high number of females in our study could be explained by the high proportion of elderly females in the South African population. ${ }^{5}$

\section{Diagnosis}

There were significant differences between the diagnostic profiles of patients admitted to this unit compared to units in developed countries. The most common diagnosis in our unit was cognitive disorders (49.5\%) followed by psychotic disorders $(36.9 \%)$. In a similar study conducted in the United States, ${ }^{19} 62.5 \%$ of inpatients had an affective disorder, whereas an Israeli study ${ }^{20}$ reported equal rates of affective disorders $(31.6 \%)$ and psychosis (32.1\%). In the Australian study, ${ }^{15}$ the most common primary diagnosis was affective disorders (39\%) followed by dementia (27.5\%) and psychotic disorders (25.8\%). The differences in the diagnostic profiles of these units are probably not due to a higher prevalence of neurocognitive and psychotic disorders in less-developed countries but rather due to the limited resources available and a shortage of appropriate caring facilities and social support for patients in developing countries. ${ }^{5,13}$ It also emphasises that our unit is only able to admit the most severe cases of behaviourally disturbed and disruptive patients and is therefore unable to provide care for patients with affective disorders that may warrant admission.

There was a high rate of substance misuse in our population: $10.8 \%$ of the patients in our study were diagnosed with substance-related disorders. This is higher than in similar international studies ${ }^{15}$ and emphasises that substance misuse is a noteworthy concern in older patients.

The prevalence of medical comorbidities in this study was high (74.5\%). People with psychiatric illness generally have higher rates of medical comorbidity compared to the general population for various reasons.

These include both patient factors (such as unhealthy behaviours, poor adherence to treatment and limited helpseeking and attendance of appointments) and healthcare factors (fewer investigations and less availability and quality of medical advice). ${ }^{21}$ This becomes even more pronounced with advanced age.

Elderly patients with mental disorders had more medical comorbidities than those without mental disorders, and these comorbidities are often detected late and inadequately managed..$^{22}$ Common medical comorbidities in this study included hypertension, hypercholesterolemia and diabetes mellitus. Considering the high rates of cardiovascular risk factors of patients in this group, the high number of patients with a vascular dementia is, perhaps, unsurprising.

Multiple patients had a documented Vitamin B12 deficiency (9.8\%). A local study found a similar prevalence of Vitamin B12 deficiency in the general elderly population. ${ }^{23}$ Although the aetiology of Vitamin B12 deficiency is multifactorial, the high number of patients with Vitamin B12 deficiency could be explained by the poor nutritional status of elderly people in South Africa. ${ }^{23}$ 


\section{Limitations and recommendations}

This study involved patient data collected over a period of 3 years, which provides a comprehensive overview of patients admitted to the unit. However, the study was a retrospective record review and therefore depended on the quality of the information in the patients' folders. Diagnoses were made by the clinical team according to the DSM IV-TR but were not generated with standardised assessment instruments. The treating clinician may have had difficulty distinguishing the primary and comorbid diagnoses, as there is often an overlap in symptomatology and aetiological factors of psychiatric disorders.

\section{Conclusion}

The findings of this study illustrate that the single psychogeriatric inpatient unit in the Western Cape Province is only able to admit high-risk patients with severe mental illness and multiple comorbidities. This sole unit is therefore not sufficient to meet the needs of aged mentally ill patients in the province. The study emphasises the need for the restructuring of resources, further research, and the implementation of other strategies (such as intensive outpatient programmes and other specialised services), which may decrease the frequency of admissions to inpatient geriatric units. The number of these specialised units should also be increased - not only in the Western Cape but also in the rest of the country.

\section{Acknowledgements}

The authors acknowledge Corion Jordan at Stikland Hospital for her assistance with the collection of the data used in this study.

\section{Competing interests}

The authors have declared that no competing interests exist.

\section{Authors' contributions}

D.P.A. collected all data and wrote the manuscript. E.G. critically revised and co-wrote the manuscript. D.J.N. and L.K. conceptualised the project and critically revised the manuscript. F.P. contributed to the final version of the manuscript.

\section{Funding Information}

This research received no specific grant from any funding agency in the public, commercial or not-for-profit sectors.

\section{Data availability statement}

Data sharing is not applicable to this article as no new data were created or analysed in this study.

\section{Disclaimer}

The views and opinions expressed in this article are those of the authors and do not necessarily reflect the official policy or position of any affiliated agency of the authors.

\section{References}

1. World Health Organization. Ageing and health - Fact sheet [homepage on the Internet]. World Health Organization Media Centre; 2015 [cited 2018 Jan 02]. Available from: http://www.who.int/mediacentre/factsheets/fs404/en/.

2. World Health Organization. Mental health of older adults - Fact sheet [homepage on the Internet]. World Health Organization Media Centre;
2017 [cited 2018 Jan 02]. Available from: http://www.who.int/mediacentre/ factsheets/fs381/en/.

3. Reynolds CF, Cuijpers P, Patel V, et al. Early intervention to reduce the global health and economic burden of major depression in older adults. Ann Rev Public Health. 2012;33(1):123-135.

4. Lima CA, Leibing A, Buschfort R. Ageing and mental health resources for older persons in the African region of the World Health Organization. S Af J Psychiatr. 2008;14(1):8-11. https://doi.org/10.1146/annurev-publhealth031811-124544

5. Statistics South Africa. Census 2011: Profile of older persons in South Africa. Statistics South Africa. Pretoria; 2014

6. Herman AA, Stein DJ, Seedat S, Heeringa SG, Moomal H, Williams DR. The South African Stress and Health (SASH) study: 12-month and lifetime prevalence of common mental disorders. S Afr Med J. 2009;99(5):339-344.

7. Olayinka OO, Mbuyi NN. Epidemiology of dementia among the elderly in subSaharan Africa. International Journal of Alzheimer's Disease. 2016.

8. De Jager CA, Msemburi W, Pepper K, Combrinck MI. Dementia prevalence in a rural region of South Africa: A cross-sectional community study. J Alzheimer Dis. 2017;60(3):1087-1096. https://doi.org/10.3233/JAD-170325

9. Van der Poel R, Heyns P. Algorithmic case prediction in relation to local clinician diagnosis in an indigenous South African population. In: 27th Internationa Conference of Alzheimer's Disease International (ADI). London; March 2012.

10. Ben-Arie O, Swarz L, Teggin A, Elk R. The coloured elderly in Cape Town A psychosocial, psychiatric and medical community survey. Part II. Prevalence of psychiatric disorders. S Afr Med J. 1983;64(27):1056-1061.

11. Ramlall S, Chipps J, Pillay B, Bhigjee A. Mild cognitive impairment and dementia in a heterogeneous elderly population: Prevalence and risk profile. Afr J Psychiatr. 2013;16(6):456-465. https://doi.org/10.4314/ajpsy.v16i6.58

12. Kalula SZ, Ferreira M, Thomas KGF, De Villiers L, Joska JA, Geffen LN. Profile and management of patients at a memory clinic. S Afr Med J. 2010;100(7):449. https://doi.org/10.7196/SAMJ.3384

13. Van Heerden S, Uwakwe R, Potochnick F. The future of old age psychiatry in Africa. Afr J Psychiatry. 2011;14(5):343-345. https://doi.org/10.4314/ajpsy.v14i5.1

14. Statistics South Africa. Community survey 2016 statistical release. Pretoria: Statistics South Africa, 2016; p. 107.

15. Smith B, Hassett A, Harrigan S, Fortune T. A profile of inpatient admissions to an aged psychiatry service in Victoria. Australas Psychiatry. 2010;18(2):146-151. https://doi.org/10.3109/10398560903314112

16. American Psychiatric Association. Diagnostic and statistical manual of menta disorders. 4th ed. Washington, DC: American Psychiatric Association; 2000.

17. Moss F, Wilson B, Harrington S, Ames D. Psychiatric diagnoses, outcomes and length of stay of patients admitte to an acute psychogeriatric unit. Int I Geriat Psychiatr. 1995;10(10):849-854. https://doi.org/10.1002/gps.930101006

18. Petersen I, Lund C. Mental health service delivery in South Africa from 2000 to 2010: One step forward, one step back. S Afr Med J. 2011;101(10): 751-757.

19. Yasgan IC, Greenwald BS, Kremen NJ, Strach J, Kramer-ginsberg E. Geriatric psychiatry versus general psychiatry inpatient treatment of the elderly. Am J Psychiatr. 2004;161(February):352-355. https://doi.org/10.1176/appi.ajp.161.2.352

20. Heinik J, Barak J, Salgenik I, Elizur A. Patterns of two psychogeriatric hospitalization services in Israel: A one-year survey. Int J Geriatr Psychiatry. 1995;10(12): 1051-1057. https://doi.org/10.1002/gps.930101210

21. Mitchell AJ, Malone D, Doebbeling CC. Quality of medical care for people with and without comorbid mental illness and substance misuse: Systematic review of comparative studies. Br J Psychiatry. 2009;194(6):491-499. https://doi.org/ 10.1192/bjp.bp.107.045732

22. Loi S, Chiu E. Medical comorbidity in psychogeriatric patients. Curr Opin Psychiatry. 2011;24(4):355-358. https://doi.org/10.1097/YCO.0b013e3283454d9e

23. Charlton KE, Kruger M, Labadarios D, Womarans $P$, Aronson I. Iron, folate and vitamin B12 status of an elderly South African population. Eur J Clin Nutr. 1997;51(7):424-430. https://doi.org/10.1038/sj.ejcn.1600402 